\title{
Process mapping: a tool to foster intra- and inter-organizational coordination in primary care
}

\author{
SILVINA SANTANA ${ }^{1, A, ~ B, ~ D-G ~}$, PATRÍCIA REDONDO², A, B, D, E \\ ${ }^{1}$ Institute of Electronics Engineering and Telematics of Aveiro \& Department of Economics, Management \\ and Industrial Engineering, University of Aveiro, Portugal \\ ${ }^{2}$ Department of Economics, Management and Industrial Engineering, University of Aveiro, Portugal
}

A - Study Design, B - Data Collection, C - Statistical Analysis, D - Data Interpretation, E - Manuscript Preparation, F - Literature Search, G - Funds Collection

Summary Background. Methods and tools to support the analysis, discussion and improvement of intra- and inter-organizational processes in primary care settings are in urgent need.

Objectives. Our goal is to show how using Petri nets to map processes in health centers may improve intra- and inter-organizational coordination of care.

Material and methods. We carried out a case study on a health center located in the Centro Region of Portugal. Information was collected through direct observation, documental analysis and semi-structured interviews to relevant collaborators. Petri nets were used as a modeling tool.

Results. Generally, health center collaborators do not have a global vision of the processes in which they are involved. Process mapping has allowed one to identify the specificities of each process, sub-processes and tasks and related documentation and actors; to identify transitions of care, to define and to represent the actions, waiting times and decisions in a visual way and to promote the enlarged discussion of processes, tacit knowledge and working practice. A number of opportunities for process improvement have been identified, namely in care transitions where patient management and safety are critical.

Conclusions. Process modelling techniques are an effective tool to support better process design and implementation and more effective coordination of care intra- and inter-organizations. They may be used at several levels, to allow depicting and visualizing more or less detail in a particular process, depending on the objective of the exercise and the actors involved. Key words: process mapping, coordinated care, Petri net, care transition, health center, primary care.

Santana S, Redondo P. Process mapping: a tool to foster intra- and inter-organizational coordination in primary care. Fam Med Prim Care Rev 2018; 20(1): 41-46, doi: https://doi.org/10.5114/fmpcr.2017.72150.

\section{Background}

The health center $(\mathrm{HC})$ is a key building block of the Portuguese National Health Service. Therefore, the quality of the work processes running there and of the transitions of care maintained with other entities of the health and social care system is crucial. In fact, the health of each individual and of the population in general depend on them, as well as the satisfaction and safety of users and the efficiency with which the $\mathrm{HC}$ attends to the needs of the population. Subjacent to these outcomes are aspects such as quality of medical care and of services in general. Fundamentally, they all depend to a great extent on the quality of the several processes being implemented.

Considerable attention is now being placed on modelling health care processes and developing information systems to support intra- and inter-organizational care process activation and coordination [1, 2]. However, the use of modelling techniques in the health sector is still much less frequent than in other fields, such as computer networks and production systems [3]. On the other hand, the information available about health care processes in $\mathrm{HC}$ in Portugal is scarce. Therefore, mapping health care processes in $\mathrm{HC}$, by identifying, understanding and documenting the tasks performed and the conditions under which they are implemented, is of paramount importance: what tasks are being executed and when, who performs each task and which other resources are used in its execution, how the tasks are aligned and executed in time and how they interact with each other, which documents are associated to them [4]? Process mapping allows these issues to become explicit and visible $[5,6]$. In the logic of care continuity, it is also important to understand how the $\mathrm{HC}$ relates with other entities.

A process may be defined as a group of logically interconnected tasks using organizational resources to generate predefined results in order to support its strategy and its objectives $[7,8]$. A process has inputs, outputs, time, space, order, objectives and values which, when logically interrelated, will result in a structure to deliver a product [8]. Each organization maintains a set of more or less explicit processes, e.g. those related to the delivery of services, marketing, product innovation and management of human resources [9]. Process mapping is a very useful and powerful technique to detail organizational processes, exposing aspects that influence their performance.

There are several methods and techniques available to analyze, model, publish, optimize and control processes; however, many are informal, in the sense that the diagrams used do not possess a pre-defined semantic [5]. The resulting models are usually very intuitive, and their interpretation depends on the person developing them, the domain of application and the characteristics of the process being analyzed.

In this study, we use Petri nets as a modelling tool, as they can describe, in an abstract manner, the specificities of a process [10]. Developed by Carl Petri in 1962, Petri nets have the great advantage of allowing for the graphical representation of processes, immensely facilitating the sharing of information in its different phases [11]. Three main reasons justify the application of Petri 
nets: they have formal semantic and graphical representation; they are able to clearly model the states of a process; they present the diversity and availability of analytic techniques [7].

Petri nets offer promising support to the modelling, analysis and simulation of discrete systems and events and allow for the modelling and visualization of diverse concepts and relationships, namely parallelism and competition, sharing of resources, synchronization, memorization and limitation of resources. During the first efforts to model a complex process, Petri nets may be of vital help by making the knowledge available and the practice explicit. As they allow one to work at several levels of abstraction, Petri nets may be adopted as a working tool by heterogeneous and interdisciplinary groups, fostering the sharing of information, the inclusion of diverse perspectives and the validation of the process by different intervening parties. The resulting diagrams make it easier to detect problematic points and to rethink their execution. In extreme situations, Petri nets may contribute to the reengineering of a process, with significant gains in terms of execution and waiting times, quality of work done and satisfaction of intervening agents. When the final goal is the digitalization of part or the whole of a process, Petri nets are the first step toward the implementation of a workflow management system.

\section{Objectives}

The goal of this work is to show how Petri nets may be used in primary care to map processes that involve a patient/client, including those shared with external entities, to find and visualize problematic points in the process and to foster further discussion and shared understanding. We use one process identified in a case study conducted in a $\mathrm{HC}$ modelled at a level of detail suitable for the demonstration of the technique and discuss the benefits that may arise from its use.

\section{Material and methods}

\section{Study design}

We sought to concisely describe the purpose of the processes and the functional areas involved, as well as sub-processes, tasks, timings, responsibilities and documents/forms used: what was done, who intervened in the process, when and where were tasks executed, which documents were associated with each task within a particular process?

The research is exploratory, given the scarcity of studies on healthcare processes in Portugal. The HC is understood as a complex reality, given the characteristics of the work developed there and the diversity of entities involved. A single organization was analyzed as the primary case study in order to generate knowledge about the phenomenon [12].

Two health centers in the District of Aveiro were selected, one to run the pilot study in the preparatory phase and the other to run the main study. They were chosen because they represented well the "health center" entity in Portugal, delivering most or all of the services expected from similar organizations. One health center was hosting the Regional Coordinating Team of the National Network of Long Term Integrated Care (RNCCl - Rede Nacional de Cuidados Continuados Integrados), providing the opportunity to study a number of interactions with other entities in the network, and this was the reason why it was chosen to run the main case study.

\section{Basic concepts in Petri nets}

An exhaustive discussion of Petri nets is not within the scope of this paper. We briefly introduce the subject to enable the reader to follow the discussion.

A Petri net can be shown in a diagram composed of two basic elements: the transition, the active element of the network, corresponds to the action, a task that is performed by an actor, and is represented by a rectangle; the place, the passive element of the network that characterizes a condition, does not change the state of the network and is represented by a circle [7]. Conditions assure that tasks are performed in the right order and allow one to verify the state of the case being processed [11]. Figure 1 shows a simple Petri net illustrating these basic concepts.

Places may contain tokens, as illustrated by the small black circle in Figure 1 . Tokens represent entities and may be of a physical nature, such as a patient, or information. The lines connecting transitions and places are named arcs. In a Petri net, the structure of the network is fixed, and only the position of the token changes. For example, to change the starting condition, the transition Task 1 must take the token in $\mathrm{C} 1$ and put it in C2.

Petri nets are easy to use, have a strong mathematical basis and a number of analytical techniques are available for them. Nevertheless, the classical Petri net has shortcomings in many practical situations, becoming too large and inaccessible, or not able to model a particular activity. Therefore, a number of functionalities have been added in so-called high-level Petri nets, namely the color extension, time and hierarchy. The color extension enables the connection of object characteristics (e.g. patient name) with its representation in the network, i.e. the corresponding token. The time extension allows one to introduce information related to time in the network. Tokens receive a timestamp and a value that specify the time from which the token is available (e.g. consultation hour). The enabling time of a transition is the earliest moment at which its input places contain sufficient available tokens, e.g. the hospital doctor cannot discharge the patient (physical token) waiting to go home if the results from his blood tests and echography (tokens representing patient information) are missing at the point of care. The hierarchical extension allows one to add structure to the network. It is denoted by a double-bordered square, as in Task 1 in Figure 1, representing a subnetwork comprising places, transitions, arcs and sub-processes in itself. Because a process can be constructed from sub-processes, which in turn can also be constructed from further sub-processes, it is possible to structure a complex process hierarchically.

There may be tasks in a process that are only executed for a number of cases. The order in which tasks are performed may also vary from case to case. By routing a case along a number of tasks, we can determine which tasks need to be carried out and in which order. Four basic constructions for routing are avail-

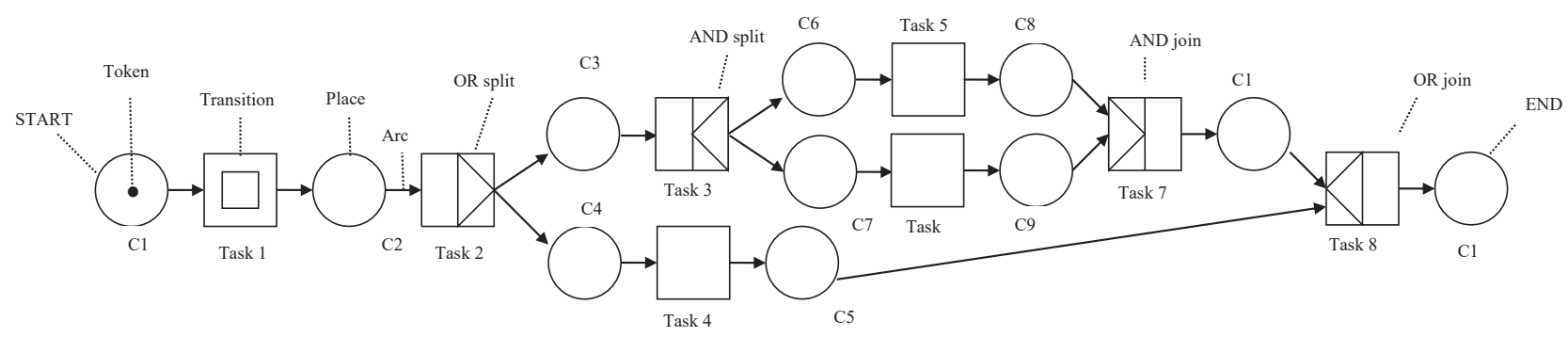

Figure 1. A Petri net showing basic building elements 
able. In sequential routing, tasks are performed one after the other; the completion of the first task is required before the second may happen. In parallel routing, more than one task may be executed simultaneously; the expressions AND-split (about to start more than one task simultaneously), such as in Task 3 in Figure 1, and AND-join (task synchronization), such as in Task 7 in Figure 1, are used to indicate such possibility. When routing in a process is based upon the attributes of a particular case, it is said to be selective; there are several options of execution available in the parallel paths, but only one may be selected, with OR-split separating the alternatives (such as in Task 2 in Figure 1) and OR-join joining the several alternative paths (such as in Task 8 in Figure 1). Finally, when a task has to be performed more than once, we call this form of routing iteration; it is built from OR-split, and it is not depicted in Figure 1 for the sake of space and clarity.

\section{Measures}

The protocol for the semi-structured interviews was designed along the following themes: existing processes; tasks executed; objectives of processes and respective tasks; actors responsible for the execution of tasks and conditions under which they were executed; sequencing of all tasks, execution timing and restrictions to execution; documents associated to each task and information resources involved, including software applications and forms. Questions covering these themes were always placed in the same order: First - questions related to the area being researched, including the objectives of the main macro activities in which the $\mathrm{HC}$ was involved; Second - questions related to processes, sub-processes and tasks, in order to obtain detailed knowledge about the processes; Third - questions about the actors intervening in the processes and the flow of information; Fourth - questions related to documents used; Finally - questions about transitions of care with external entities.

\section{Procedures}

Given the complexity of health centers, the fact that these are entities completely dependent from the State and issued legislation, the scarcity of information published on the subject under analysis and the expected low level of variation regarding generic work practice in health centers countrywide, we conducted a pilot case study in a similar organization, in order to better prepare the main field intervention, by observing work practice, analyzing work documents and software applications and interviewing health professionals. We chose two health centers in the same region, but sufficiently apart in order to avoid contamination, similar in geographic and socio-economic environment, organizational structure, services provided, protocols and documents used and work force. All health centers in Portugal use the same information systems for doctors (SAM), nurses (SAPÉ) and management of administrative work (SINUS); therefore, a degree of standardization imposed by these applications was expected. The pilot included interviews with five professionals: an administrative assistant, an administrative section manager, a social worker, a nurse and a family doctor, as participants in several of the processes in place and representing the main professional groups. The pilot case study allowed us to understand in detail how an $\mathrm{HC}$ is usually structured and run in Portugal and to validate and refine the protocol and tools to be used to collect information for the main study, namely by adding specific questions to cover the specificities of each type of process and subprocess. The main conclusion from the pilot study was that many processes were not described nor formalized, clearly confirming the relevance and timeliness of the study.

The main case study began in 2011, and the information was reconfirmed during the first semester of 2014 to check if the processes, as described in our study, had been maintained after the structural changes that were being introduced in primary care in Portugal already in 2011. Several visits were performed in order to observe daily functioning, collect documents, study the operation of information systems and conduct the interviews. We interviewed fifteen professionals in order to cover all types of activities with which they were specifically involved, including: three family doctors, seven nurses, two administrative workers, a nutritionist, a dental hygienist and a social worker. The interviews were conducted individually and recorded with the consent of each respondent.

In this study, we describe one of the identified processes. The description is according to the following structure: objective, intervening actors, entity processed, conditions and rules of execution, diagram. The description of the supporting tables and the documental system developed is not within the scope of this paper. The conditions were those in place at the time of information collection.

\section{Results}

\section{General results}

Generally, $\mathrm{HC}$ collaborators did not have a global view of the processes in which they were involved, focusing very much on the activities they usually performed and showing difficulties in systematizing and describing the broader processes where those activities were embedded. Despite the questions raised, shared understanding and a consensus was reached on processes involving interaction with patients/external clients (Table 1).

\begin{tabular}{|c|c|}
\hline Process & Most important sub-processes \\
\hline \multicolumn{2}{|l|}{ Schedule a consultation } \\
\hline Admission of patient & Registration in a consultation \\
\hline \multirow[t]{15}{*}{ Consultation } & Public health consultation \\
\hline & Maternal health consultation \\
\hline & Infant health consultation \\
\hline & Family planning consultation \\
\hline & Adult consultation \\
\hline & Diabetes consultation \\
\hline & Open access consultation \\
\hline & Telemedicine consultation \\
\hline & Oral hygiene consultation \\
\hline & Nutrition consultation \\
\hline & Social service consultation \\
\hline & Adolescent consultation \\
\hline & Antismoking consultation \\
\hline & Alcohology consultation \\
\hline & $\begin{array}{l}\text { Pulmonology Diagnostic Center } \\
\text { consultation }\end{array}$ \\
\hline \multicolumn{2}{|l|}{ Schedule a home visit } \\
\hline \multirow[t]{4}{*}{ Home visit } & Medical home visit \\
\hline & Nurse home visit \\
\hline & Postpartum home visit \\
\hline & Social service home visit \\
\hline \multicolumn{2}{|l|}{ Health education } \\
\hline \multirow[t]{4}{*}{ Nursing intervention } & Vaccination \\
\hline & $\begin{array}{l}\text { Psychoprophylactic preparation for } \\
\text { childbirth }\end{array}$ \\
\hline & Nursing treatment \\
\hline & Distribution of methadone \\
\hline \multicolumn{2}{|l|}{ Discharge of patient } \\
\hline \multicolumn{2}{|l|}{$\begin{array}{l}\text { Handling patient within } \\
\text { the RNCCI }\end{array}$} \\
\hline Handling claim & \\
\hline
\end{tabular}




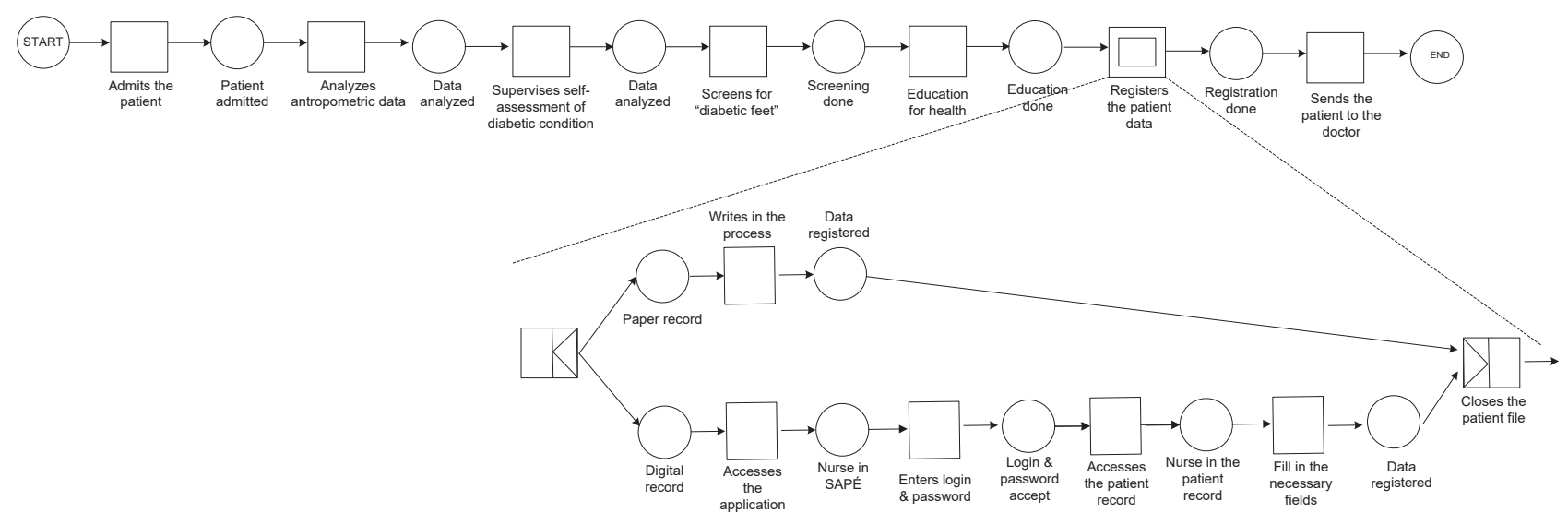

Figure 2. Nurse consultation

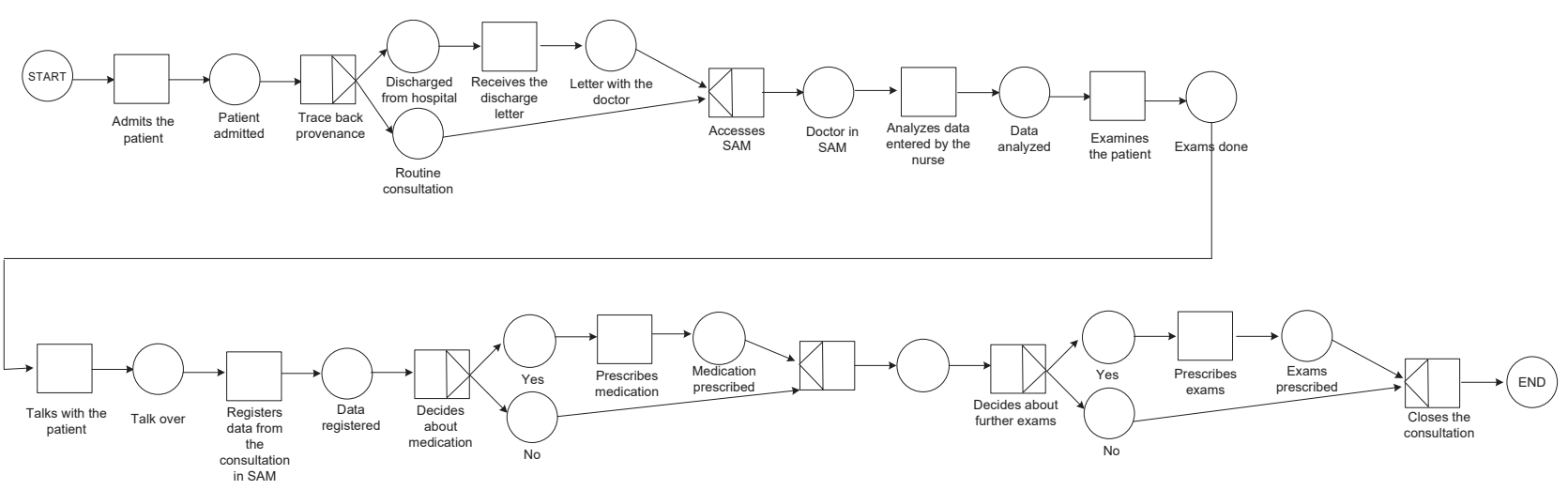

Figure 3. Doctor consultation

The HC was interacting with various entities: health centers, hospitals, RNCCl units, family health units, universities, the Portuguese League Against Cancer, the Social Security, police and fire departments, schools, parish councils, the municipality, entities related to specific programs such as drug addiction, private non-profit institutions of social solidarity, which in Portugal are the main providers of social care and home care. Each type of relationship involved specific processes with varied levels of formalization and documentation and, many times, transitions of care that proved to be challenging as they were not supported by information systems and would involve processes without any embedded control mechanism.

\section{Sub-process "Diabetes consultation"}

Objective: Early diagnosis of the problem, illness prevention, prescription of treatment and promotion of healthy lifestyles.

Intervening actors: user/patient, family members or other caregivers and all the collaborators of the $\mathrm{HC}$ intervening in the process.

Entity processed: the token in this process represents the user/patient; the collaborators executing the tasks are a family doctor and a nurse.

Conditions and rules of execution of tasks within the process: a diabetes consultation is composed of two major sub-processes: nursing consultation and doctor consultation. A routine consultation is held every three months and is always scheduled from one consultation to the next. It always starts with a nursing consultation. The nurse educates the patient on dietary habits, measures their blood pressure and waist circumference, calculates their body mass index, controls for self-monitoring of diabetic condition, and examines their feet once a year to check for wounds. Afterwards, the user meets the family doctor, who analyzes the data collected by the nurse and performs own tests in order to assess the status of the user and to prescribe ap- propriate treatment and exams if necessary. The consultation is computerized in both SAM and SAPÉ. When the user comes to the $\mathrm{HC}$ for a consultation after being discharged from the hospital, she/he brings a letter of discharge from the hospital doctor and a letter of discharge from the hospital nurse, occasionally a third letter from the hospital social worker. In exceptional cases, a hospital doctor calls a family doctor over phone. When a family doctor decides to send a patient to a specialist for further exams, they give the patient a referring letter to be presented to the specialist. Figures 2 and 3 represent the corresponding Petri nets at different abstraction levels.

\section{Discussion}

The study allowed us to demonstrate the feasibility and advantages of using process mapping techniques in the context of an important entity of the Portuguese National Health Service, the health center, to help improve care coordination in primary care. This is a pioneer study, and no similar effort was found in literature at the time of execution, as work using this technique is usually directed to the development of workflow management systems or decision support systems, with an emphasis on information systems and technologies and software development [3].

The first conclusion is that it is relatively easy to introduce a layperson, such as family doctors, nurses and administrative personnel, to the basic aspects of Petri nets, so that they can collaborate on understanding and improving the processes in which they participate. We have identified and mapped ten main processes involving a patient or other client (e.g. family doctor, nurse, teachers and students in a public health initiative), as well as the most important sub-processes, including all types of consultations, home visits and nursing interventions.

The informality of many processes became evident, materialized by the difficulty some professionals have shown in de- 
scribing how work was being done in a systematic and consistent way and the need they felt to create their own tools to deal with user information. Activities were being guided by formalisms imposed by law and regulations and by the best practice of professional groups. However, some of the situations were not framed by these formalisms, and processes involving transitions of care with external entities are clear examples of areas where improvement could be achieved.

Some processes were not standardized and represented in the computerized information system, and applications were not completely integrated: administrative staff, doctors and nurses were working with different platforms, for which in some cases (e.g. diabetes consultation) connections had been created only recently. While many types of consultation were already computerized, some were still not represented in the computer systems, and activities were being processed manually. This situation created some difficulties at different levels, as well as possible discontinuities in the provision of care, due to inefficient communication between providers. For example, for a user referred by a doctor to social service consultation, the user personal file should be available for the social worker to register the diagnosis. Accordingly, the relevant parts of the clinical and social history of the user defined as public would be accessible in a controlled manner to all professionals intervening in the treatment. However, by the time we collected the data, no documents pertaining to each case were being shared between the doctor and social worker, and the social service consultation was one of those still not handled by the information systems. Therefore, it was up to the social worker to make the decision to call the doctor and provide information concerning the best interest of the patient; otherwise, the doctor would remain unaware of any social issues that might compromise the treatment.

Communication with external entities was mostly done by phone, paper and face-to-face. SAM and SAPÉ were introduced in health centers and hospitals in Portugal many years ago, but at the time of the study, there was still no connection implemented between hospital and health center versions of the systems. Reliance on unstructured relationships and communications based mostly on telephone and personal contacts could therefore be the cause of problems at the process level. For example, on transitions from hospital care, the information exchanged between care providers is that contained in the discharge letters patients must bring to the consultation with family doctor and nurse. Only in some situations might there have been a previous call from the hospital. Family doctors also used a letter delivered by the patient to send information to specialist when referring a patient to another level of care. In relationships with other entities, including partnerships for health education, the contacts were also mostly rather informal. The most critical situation was the transition of a user from the ambulance

Source of funding: This work was funded by the authors' own resources. Conflict of interest: The authors declare no conflict of interests.

\section{References}

1. Macris A, Papakonstantinou D, Malamateniou F, et al. Using ontology-based knowledge networks for user training in managing healthcare processes. Int J Technology Management 2009; 47(1/2/3): 5-21.

2. Zacarias M, Caetano A, Pinto S, et al. Modeling Organizations: The "Operating Systems" metaphor. 2005. Available from URL: http:// www.inesc-id.pt/ficheiros/publicacoes/2425.pdf.

3. Salimifard K, Hosseini S, Moradi M. Improving Emergency Department Processes Using Coloured Petri Nets. In: Moldt D, ed. International Workshop on Modeling and Business Environments (ModBE'13); June 24; Milano, Italy; 2013: 335-349.

4. Von Rutte R. Managing processes. The TQM Magazine 1990; 2(6), doi: doi.org/10.1108/EUM0000000003053.

5. Santos C, Ferreira C, Tribolet J. Modelação e verificação formal de processos de negócio em ambiente hospitalar. 7a Conferência da Associação Portuguesa de Sistemas de Informação. Universidade de Aveiro; 2007 (in Portuguese).

6. Tribolet J, Macedo P. Modelação de processos de produção em engenharia organizacional. 5 o Conferência da Associação Portuguesa de Sistemas de Informação. Lisboa; 2004 (in Portuguese).

7. Aalst W, Hee K. Workflow management models, methods and systems. Cambridge (MA): The Mit Press; 2002. service provider, usually fire departments, to the HC. Indeed, no form was being used by the transportation service or the $\mathrm{HC}$ to register such a situation, and no report was being made possible events affecting the patient during transportransportation services by ambulance to the $\mathrm{HC}$ were not paid would need a document signed by a doctor, i.e. a document ment of costs from the National Health Service. This suggests these processes involving care transitions with external enimprovement could possibly be achieved by considering health related aspects and designing the processes around the needs principal users, i.e. patients and healthcare providers. rated in a countrywide web-based platform. The entire course of the patient, from being discharged from the hospital and detailed data on personal, clinical and social aspects, i.e. the bio-psycho-social profile, was made available to the authorized staff of health and social care institutions participating in the network in a controlled manner. Although there was no workthis connectivity of information systems allowed for the streamlining of most tasks related to the management of patients within the network. However, the RNCCI platform was not intewith other information systems in use in the $\mathrm{HC}$ and did discharged from the network had to reassume their traditio role of connector between levels of care, delivering the doctor, nurse and sometimes social worker discharge letters from the rehabilitation unit to the family doctor in the $\mathrm{HC}$.

\section{Conclusions}

This case study has demonstrated the viability and the benefits of using process mapping techniques in the context of Analyzing processes in health centers may bring innovation to primary care, making it possible to introduce improvement at ing how activities are conducted at the micro level and how to operationalize the relationships with other institutions may be an important step toward the reconfiguration and the sustainin Portugal. by the National Health Service. This seems to be confirmed by the fact that transport by ambulance from the $\mathrm{HC}$ to the hospi- 
8. Villela C. Mapeamento de processos como ferramenta de reestruturação e aprendizado organizacional. Florianopolis: Universidade Federal de Santa Catarina; 2000 (in Portuguese).

9. Hindle J. Understanding business processes. Health Manpow Manage 1997; 23(4-5): 181-183.

10. Prickett P. A Petri-net based machine tool maintenance management system. Industrial Management \& Data Systems 1997; 97(4): 143-149, doi: https://doi.org/10.1108/02635579710173211.

11. Dallavalle de Pádua S, Yoshizawa da Silva A, Porto A, et al. O potencial das redes de Petri em modelagem e análise de processos de negócio. Gestão \& Produção 2004: 11(1): 109-119 (in Portuguese).

12. Yin R. Case study research: Design and methods. $2^{\text {nd }}$ ed. Beverly Hills (CA): Sage Publishing; 1994.

Tables: 1

Figures: 3

References: 12

Received: 11.08 .2017

Reviewed: 24.08 .2017

Accepted: 12.10 .2017

Address for correspondence:

Silvina Santana, Professor of Business Management Sciences

Institute of Electronics Engineering and Telematics of Aveiro

Department of Economics, Management and Industrial Engineering

Research Unit in Governance, Competitiveness and Public Policies

University of Aveiro

Campus de Santiago

3810-193 Aveiro

Portugal

Tel.: +351 234370840

E-mail: silvina.santana@ua.pt 